\title{
ON THE TIME DECAY OF DIFFUSION WAVES FOR A CLASS OF PARABOLIC SYSTEMS OF CONSERVATION LAWS
}

\author{
Paulo R. Zingano*
}

\begin{abstract}
We derive time decay rates in $L^{2}$-norm for solutions to the Cauchy problem of the parabolic system

$$
\boldsymbol{u}_{t}+(\boldsymbol{u} \varphi(|\boldsymbol{u}|))_{x}=\left(B(\boldsymbol{u}) \boldsymbol{u}_{x}\right)_{x}
$$

and its $N$-dimensional analoguc

$$
\boldsymbol{u}_{t}+\sum_{k=1}^{N} \frac{\partial}{\partial x_{k}}\left(\boldsymbol{u} \varphi_{k}(|\boldsymbol{u}|)\right)=\sum_{k=1}^{N} \frac{\partial}{\partial x_{k}}\left(B^{[k]}(\boldsymbol{u}) \frac{\partial \boldsymbol{u}}{\partial x_{k}}\right)
$$

provided only that the initial state $\boldsymbol{u}(\cdot, 0)$ is in $I_{t}^{1} \cap L^{2}$, but otherwise arbitrary. Ilere, $\boldsymbol{u}=\left(u_{1}, \ldots, u_{m}\right)$ is the vector of unknowns, $\varphi, \varphi_{1}, \ldots, \varphi_{k}$ are given scalar functions, and $B, B^{[1]}, \ldots, B^{[N]}$ are 11 niformly positive definite matrices of order $m$ whose off-diagonal elements $b_{i j}(u)$ are bounded in terms of $a_{i}$. The mothod is based on energy estimates and can be adapted to other problems. The decay rates obtained are optimal.
\end{abstract}

\section{Resumo}

São derivadas taxas de decaimento na norma $L^{2}$ para as soluçõos do problema de Cauchy do sistema parabólico

$$
\boldsymbol{u}_{t}+(\boldsymbol{u} \varphi(|\boldsymbol{u}|))_{x}=\left(B(\boldsymbol{u}) \boldsymbol{u}_{x}\right)_{x}
$$

e do problema correspondente em $N$ dimensöes

$$
\boldsymbol{u}_{i}+\sum_{k=1}^{N} \frac{\partial}{\partial x_{k}}\left(\boldsymbol{u} \varphi_{k}(|\boldsymbol{u}|)\right)=\sum_{k=1}^{N} \frac{\partial}{\partial x_{k}}\left(B^{[k]}(\boldsymbol{u}) \frac{\partial \boldsymbol{u}}{\partial x_{k}}\right)
$$

${ }^{*}$ This work was supported by CNPq (PQ 301236/91, AVG 453835/96-1) and FAPERGS (APC 96/1696-6) 
assumindo-sc que o estado inicial $u(\cdot, 0)$ pertence ao espaço $L^{1} \cap L^{2}$, sendo possivelmente grande. Nas cquações acima, $u=\left(u_{1}, \ldots, u_{m}\right), \varphi, \varphi_{1}, \ldots, \varphi_{k}$ são funç̧óss escalares dadas, c $B, B^{[1]}, \ldots, B^{[N]}$ denotam matrizes definidas positivas de ordem $m$ cujos clementos $b_{i j}(u)$ fora da diagonal são limitados cm termos de $u_{i}$. O mćtodo é bascado em cstimativas de cnergia c pode ser adaptado para outros problemas. As taxas de decaimento obtidas são optimais.

\section{Introduction}

Wo will cstablish in this article the time-asymptotic decay in $L^{2}$-norm of solutions to the initial-value problem

$$
\begin{gathered}
\boldsymbol{u}_{t}+(\boldsymbol{u} \varphi(|\boldsymbol{u}|))_{x}=\left(B(\boldsymbol{u}) \boldsymbol{u}_{x}\right)_{x} \\
\boldsymbol{u}(\cdot, 0) \in L^{1}(\mathbf{R}) \cap L^{2}(\mathbf{R})
\end{gathered}
$$

and some of its generalizations, where $\boldsymbol{u}(x, t)=\left(u_{1}(x, t), \ldots, u_{m}(x, t)\right)$ stands for the vector of unknown quantities, $|\boldsymbol{u}|$ is the Euclidean norm of $\boldsymbol{u}, \varphi$ is a contimously differentiable scalar function and $B(\boldsymbol{u})$ is an $m \times m$ uniformly positive definite matrix, i.c.,

$$
(\boldsymbol{\xi}, B(\boldsymbol{u}) \boldsymbol{\xi}) \geq \mu|\boldsymbol{\xi}|^{2} \quad \forall \boldsymbol{\xi} \in \mathbf{R}^{m}
$$

for all $\boldsymbol{u}$ concerned, where $\mu$ is a positive constant and $(\cdot, \cdot)$ denotes the standard immer product in $\mathbf{R}^{m}$. We also assume that the of -diagonal entries $b_{i j}(\boldsymbol{u})$ of $B(\boldsymbol{u})$ salisfy, for all $\boldsymbol{u}=\left(u_{1}, \ldots, u_{m}\right)$ concerned,

$$
\left|b_{i j}(\boldsymbol{u})\right| \leq C\left|u_{i}\right| \quad \forall i, j=1, \ldots, m, i \neq j
$$

for some constant $C>0$. For basic properties of (1), we refer the reader to [11] and references therein. One example is given by the class of rotationally invariani. systems considered in [6], [13], mamely

$$
\boldsymbol{u}_{+}+\left(\boldsymbol{u}|\boldsymbol{u}|^{2}\right)_{x}=\mu \boldsymbol{u}_{x x}
$$

where $\mu$ is a positive constant. 'I'he inviscid form of equation (4) was considered in 1979 by Keylity and Kramer in comection with the elastic string problem in 
elasticity [9]. 'This system has also been studied in one-dimensional multiphase flow [7], [12], magnetohydrodynamics [1], [5] and more generally in continuum mechanics as a basic model for the propagation of plane waves in isotropic, multidimensional systems [1], [2]. For these and all systems of the form (1)-(3), we will show in Section 2 that

$$
\|\boldsymbol{u}(\cdot, t)\|_{T_{i}^{2}(\mathbf{R})} \leq C(1+t)^{-1 / 1}
$$

for all $t>0$, where the constant $C$ depends on the magnitude of $\|\boldsymbol{u}(\cdot, 0)\|_{L^{1}(\mathbf{R})}$ and $\|\boldsymbol{u}(\cdot, 0)\|_{L^{2}(\mathbf{R})}$, the dimension $m$ and $\mu>0$ given in (2) above. Thus, the solution $\boldsymbol{u}=\mathbf{0}$ of (1)-(3) is asymptotically stable under arbitrarily large disturbances; provided only that they belong to $L^{1}(\mathbf{R}) \cap L^{2}(\mathbf{R})$. The same decay behavior in the $L_{i}^{2}$-nom has been shown to hold for general systems of conservation laws

$$
\boldsymbol{u}_{t}+\boldsymbol{f}(\boldsymbol{u})_{x}=\left(B(\boldsymbol{u}) \boldsymbol{u}_{x}\right)_{x}
$$

with an arbitrary viscosity matrix $B(\boldsymbol{u})$ and flux function $\boldsymbol{f}(\boldsymbol{u})$ such that the Jacobian matrix $\boldsymbol{f}^{\prime}(\mathbf{0})$ is completely hyperbolic [3], [4], [8], under the further assumption that $\boldsymbol{u}(\cdot, 0)$ is small enough to satisly

$$
\int_{-\infty}^{+\infty}|\boldsymbol{u}(x, 0)|(1+|x|) d x+\int_{-\infty}^{+\infty}\left(|\boldsymbol{u}(x, 0)|^{2}+\left|\boldsymbol{u}_{x}(x, 0)\right|^{2}\right) d x \leq \delta
$$

for $\delta \ll 1$. In the particular case of systems verifying (1)-(3), condition (7) is not necessary and wo will show in Section 2 using a very simple argument that the estimate (5) holds for any initial state $\boldsymbol{u}(\cdot, 0)$ in $L^{\prime}(\mathbf{R}) \cap L^{2}(\mathbf{R})$, however large. If one replaces ( 1 a) by the slightly more gemeral equation

$$
\boldsymbol{u}_{t}+(\boldsymbol{u} \varphi(\boldsymbol{u}))_{x}=\left(B(\boldsymbol{u}) \boldsymbol{u}_{x}\right)_{x}
$$

then we can still derive (5) provided that $\|\boldsymbol{u}(\cdot, 0)\|_{T^{1}(\mathbf{R})}$ is sufficiently small, see Section 2. Finally, in Section 3 we will use a similar argument, to investigate the corresponding behavior for the $N$-dimensional analogue of (1),

$$
\boldsymbol{u}_{t}+\sum_{k=1}^{N} \frac{\partial}{\partial x_{k}}\left(\boldsymbol{u} \varphi_{k}(|\boldsymbol{u}|)\right)=\sum_{k=1}^{N} \frac{\partial}{\partial x_{k}}\left(B^{[k]}(\boldsymbol{u}) \frac{\partial \boldsymbol{u}}{\partial x_{k}}\right)
$$


where $\varphi_{1}, \ldots, \varphi_{N}$ are scalar functions and $B^{[1]}(\boldsymbol{u}), \ldots, B^{[N]}(\boldsymbol{u})$ are posilive definite matrices of order $m$ such that

$$
\left(\boldsymbol{\xi}, B^{[k]}(\boldsymbol{u}) \boldsymbol{\xi}\right) \geq \mu|\boldsymbol{\xi}|^{2} \quad \forall \boldsymbol{\xi} \in \mathbf{R}^{m}, k=1, \ldots, N
$$

and whose elements satisfy, for each $k=1, \ldots, N$,

$$
\left|b_{i j}^{[k]}(\boldsymbol{u})\right| \leq C\left|u_{i}\right| \quad \forall i, j=1, \ldots, m, i \neq j
$$

for all values of $\boldsymbol{u}$ in the region concerned, where $\mu, C$ are positive constants. It is then shown that

$$
\|\boldsymbol{u}(\cdot, l)\|_{L^{2}\left(\mathbf{R}^{N}\right)} \leq C(1+l)^{-N / 4}
$$

whenever $\boldsymbol{u}(\cdot, 0) \in I^{1}\left(\mathbf{R}^{N}\right) \cap I^{2}\left(\mathbf{R}^{N}\right)$, where $C$ is a positive constant, which de-

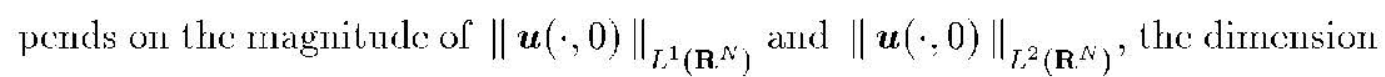
paramelers $m, N$ and $\mu>0$ given in (10). For all these problems, the decay rates given above are optimal, so that the method discussed here, in spite of its simplicity, gives sharp results. Moreover, it can be applied to other problems, see e.g. [14], [15].

As to the notation used, boldface characters will always denote vector quantities, while capital letiers will be usually reserved for matrices, with the exception of letiers $C$ and $K$, which will be used for scalar constants. A symbol like $C_{\mathcal{A}}$ represents a constant whose value depends on a set of parameters specificd by $\mathcal{A}$; we note that distinct references to the same constant symbol will not necessarily mean the same numerical value, so that we will write $2 C_{\mathcal{A}}$ again as $C_{\mathcal{A}}$, and so on. Also, we will often use subscripted variables to indicate differen1.ialion, as in $u_{t}=\frac{\partial u}{\partial t}, f(u)_{x}=\frac{\partial}{\partial x} f(u(x, t))$, and so forth. All other notation, when not standard, will be explained right after its first occurrence in the text.

\section{One-dimensional systems}

We will consider in this section the $L^{2}$ decay of solutions $\boldsymbol{u}(x, t)$ of the Cauchy problem (1)-(3) described above. The initial state $\boldsymbol{u}(\cdot, 0)$ is any Iebesgue mea- 
surable pulse with finite mass and energy, i.e., $\boldsymbol{u}(\cdot, 0) \in L^{1}(\mathbf{R}) \cap L^{2}(\mathbf{R})$, and we let $K>0$ be sufficiently large so that

$$
\|\boldsymbol{u}(\cdot, 0)\|_{T^{1}(\mathbf{R})} \leq K \quad, \quad\|\boldsymbol{u}(\cdot, 0)\|_{J^{2}(\mathbf{R})} \leq K
$$

Under these conditions, we will show below that there exists a positive comstant $C_{\mathcal{K}}$, depending on the set of parameters $\mathcal{K}=\{m, K, \mu\}$, such that

$$
\|\boldsymbol{u}(\cdot, l)\|_{J^{2}(\mathbf{R})} \leq C_{K}(1+l)^{-1 / 4} \quad \forall \ell>0
$$

We will prove this estimate in the following way. First, we note that the solution operator of (1) is $L^{1}$-contractive, i.e., we have

$$
\|\boldsymbol{u}(\cdot, l)\|_{L^{1}(\mathbf{R})} \leq\|\boldsymbol{u}(\cdot ; 0)\|_{L^{1}(\mathbf{R})} \quad \forall l>0
$$

where

$$
\|\boldsymbol{u}(\cdot, t)\|_{L^{\prime}(\mathbf{R})}=\left\|u_{1}(\cdot, t)\right\|_{I^{\prime}(\mathbf{R})}+\ldots+\left\|u_{m}(\cdot, t)\right\|_{I_{i}^{\prime}(\mathbf{R})}
$$

and similarly for $\|\boldsymbol{u}(\cdot, 0)\|_{\tau^{1}(\mathbf{R})}$. In fact, more is true: one has, for each individual component $u_{i}(\cdot, l)$ of the solution vector $\boldsymbol{u}(\cdot, l), i=1, \ldots, m$, the estimale

$$
\left\|u_{i}(\cdot, l)\right\|_{L^{1}(\mathbf{R})} \leq\left\|u_{i}(\cdot, 0)\right\|_{L^{1}(\mathbf{R})} \quad \forall \quad l>0
$$

This can be proved in a standard way as in [10], but for convenience of the reader we will briefly review the argument. Taking a regularized sign function $L_{\delta}^{\prime}$ (see e.g. [10]; [14]), we multiply the $i^{\text {th }}$ componemt of equation (1a) by $I_{s}^{\prime}\left(u_{i}(x, l)\right)$ and integrate the result over $\mathbf{R} \times[0, T]$ to get, after a few integralions by parts,

$$
\begin{gathered}
\int_{-\infty}^{+\infty} L_{\delta}\left(u_{i}(x, T)\right) d x+\int_{0}^{T} \int_{-\infty}^{+\infty} L_{\delta}^{\prime \prime}\left(u_{i}(x, t)\right) b_{i i}(\boldsymbol{u}(x, t))\left(\frac{\partial u_{i}}{\partial x}\right)^{2} d x d t= \\
=\int_{-\infty}^{+\infty} L_{\delta}\left(u_{i}(x, 0)\right) d x+\int_{0}^{T} \int_{-\infty}^{+\infty} L_{\delta}^{\prime \prime}\left(u_{i}(x, t)\right) u_{i}(x, t) \frac{\partial u_{i}}{\partial x} \varphi(|\boldsymbol{u}(x, t)|) d x d t \\
+\sum_{\substack{j=1 \\
j \neq i}}^{m} \int_{0}^{T} \int_{-\infty}^{+\infty} L_{\delta}^{\prime \prime}\left(u_{i}(x, t)\right) b_{i, j}(\boldsymbol{u}(x, t)) \frac{\partial u_{i}}{\partial x} \frac{\partial u_{j}}{\partial x} d x d t
\end{gathered}
$$


Since $L_{\delta}^{\prime \prime}\left(u_{i}\right)$ and $b_{i i}(\boldsymbol{u})$ are nonnegative, we then obtain, using (3) above,

$$
\begin{gathered}
\int_{-\infty}^{+\infty} L_{\delta}\left(u_{i}(x, T)\right) d x \leq \int_{-\infty}^{+\infty} L_{\delta}\left(u_{i}(x, 0)\right) d x+ \\
+\int_{0}^{T} \int_{-\infty}^{+\infty} L_{\delta}^{\prime \prime}\left(u_{i}(x, t)\right) u_{i}(x, t) \frac{\partial u_{i}}{\partial x} \varphi(|\boldsymbol{u}(x, t)|) d x d t \\
+C \sum_{\substack{j=1 \\
j \neq i}}^{m} \int_{0}^{T} \int_{-\infty}^{+\infty} L_{\delta}^{\prime \prime}\left(u_{i}(x, t)\right)\left|u_{i}(x, t)\right|\left|\frac{\partial u_{i}}{\partial x}\right|\left|\frac{\partial u_{j}}{\partial x}\right| d x d t
\end{gathered}
$$

where $C$ is the comstant, given in (3). Itelting $\delta \rightarrow 0$, we get (15)', since

$$
\int_{0}^{T} \int_{-\infty}^{+\infty} I_{\delta}^{\prime \prime}\left(u_{i}(x, l)\right) u_{i}(x, l) \frac{\partial u_{i}}{\partial x} \varphi(|\boldsymbol{u}(x, l)|) d x d l \rightarrow 0
$$

and

$$
\int_{0}^{T} \int_{-\infty}^{+\infty} I_{\delta}^{\prime \prime}\left(u_{i}(x, l)\right)\left|u_{i}(x, l)\right|\left|\frac{\partial u_{i}}{\partial x}\right|\left|\frac{\partial u_{j}}{\partial x}\right| d x d l \rightarrow 0
$$

by Lebesgue's Dominated Convergence 'Theorem. Another property which can be easily derived is the following energy estimate,

$$
\left\|\boldsymbol{u}\left(\cdot, T^{\prime}\right)\right\|_{L^{2}(\mathbf{R})}^{2}+2 \mu \int_{0}^{\prime}\|D \boldsymbol{u}(\cdot, t)\|_{L^{2}(\mathbf{R})}^{2} d t \leq\|\boldsymbol{u}(\cdot, 0)\|_{L^{2}(\mathbf{R})}^{2}
$$

where

and

$$
\|\boldsymbol{u}(\cdot, t)\|_{,^{2}(\mathbf{R})}^{2}=\sum_{i=1}^{m}\left\|u_{i}(\cdot, t)\right\|_{i^{2}(\mathbf{R})}^{2}
$$

$$
\|D \boldsymbol{u}(\cdot, l)\|_{L^{2}(\mathbf{R})}^{2}=\sum_{i=1}^{m}\left\|\frac{\partial u_{i}}{\partial x}(\cdot, l)\right\|_{L^{2}(\mathbf{R})}^{2}
$$

In fact, multiplying the $i^{\text {th }}$ component of equation (1a) by $u_{i}(x, t)$, integrating the result over $\mathbf{R} \times\left[0, T^{\prime}\right]$ and summing from $i=1$ to $m$, we get, after a few similar compulations,

$$
\begin{gathered}
\int_{-\infty}^{+\infty}|\boldsymbol{u}(x, T)|^{2} d x+2 \int_{0}^{T} \int_{-\infty}^{+\infty}\left(\boldsymbol{u}_{x}, B(\boldsymbol{u}) \boldsymbol{u}_{x}\right) d x d t= \\
=\int_{-\infty}^{+\infty}|\boldsymbol{u}(x, 0)|^{2} d x+\int_{0}^{t} \int_{-\infty}^{+\infty} \varphi(|\boldsymbol{u}(x, t)|) \frac{\partial}{\partial x}|\boldsymbol{u}(x, t)|^{2} d x d t
\end{gathered}
$$


from which we immediately get (16), in view of assumption (2) and

$$
\int_{-\infty}^{+\infty} p(|\boldsymbol{u}(x, l)|) \frac{\partial}{\partial x}|\boldsymbol{u}(x, l)|^{2} d x=0
$$

In order to obtain a decay rate for $\|\boldsymbol{u}(\cdot, t)\|_{T^{2}(\mathbf{R})}$, however, we need to do a more careful analysis. To this end, we multiply the $i^{\text {th }}$ component of equation (1a) by $(1+l) u_{i}(x, t)$ and integrate the result over $\mathbf{R} \times[0, T]$, which gives, summing from $i=1$ l, $\mathrm{m}$,

$$
\begin{gathered}
(1+T) \int_{-\infty}^{+\infty}|\boldsymbol{u}(x, T)|^{2} d x+ \\
+2 \int_{0}^{T}(1+t) \int_{-\infty}^{+\infty}\left(\boldsymbol{u}_{x}, B(\boldsymbol{u}) \boldsymbol{u}_{x}\right) d x d t= \\
=\int_{-\infty}^{+\infty}|\boldsymbol{u}(x, 0)|^{2} d x+\int_{0}^{T} \int_{-\infty}^{+\infty}|\boldsymbol{u}(x, t)|^{2} d x d l+ \\
+\int_{0}^{T}(1+t) \int_{-\infty}^{+\infty} \varphi(|\boldsymbol{u}(x, t)|) \frac{\partial}{\partial x}|\boldsymbol{u}(x, t)|^{2} d x d t
\end{gathered}
$$

so that, recalling (2) and (17), wc obtain

$$
\begin{aligned}
(1+ & \left.+T^{\prime}\right)\|\boldsymbol{u}(\cdot, T)\|_{L^{2}(\mathbf{R})}^{2}+2 \mu \int_{0}^{T}(1+t)\|D \boldsymbol{u}(\cdot, t)\|_{L^{2}(\mathbf{R})}^{2} d t \\
& \leq\|\boldsymbol{u}(\cdot, 0)\|_{L^{2}(\mathbf{R})}^{2}+\int_{0}^{T}\|\boldsymbol{u}(\cdot, l)\|_{L^{2}(\mathbf{R})}^{2} d t
\end{aligned}
$$

Using the elementary Sobolev inequality

$$
\left\|u_{i}(\cdot, t)\right\|_{J_{i}^{2}(\mathbf{R})} \leq C\left\|u_{i}(\cdot, t)\right\|_{l^{1}(\mathbf{R})}^{2 / 3}\left\|\frac{\partial u_{i}}{\partial x}(\cdot, t)\right\|_{h^{2}(\mathbf{R})}^{1 / 3}
$$

we get, from (13): (15):

$$
\|\boldsymbol{u}(\cdot, t)\|_{L^{2}(\mathbf{R})} \leq C_{m, K}\|D \boldsymbol{u}(\cdot, t)\|_{I^{2}(\mathbf{R})}^{1 / 3}
$$

for each $t>0$, where $C_{m, K}$ denotes a constant which depends on $m, K$. Hence, (18) yields

$$
\begin{gathered}
(1+T)\|\boldsymbol{u}(\cdot, T)\|_{L^{2}(\mathbf{R})}^{2}+2 \mu \int_{0}^{T}(1+l)\|D \boldsymbol{u}(\cdot, l)\|_{L^{2}(\mathbf{R})}^{2} d l \\
\leq\|\boldsymbol{u}(\cdot, 0)\|_{L^{2}(\mathbf{R})}^{2}+C_{m, K} \int_{0}^{T}\|D \boldsymbol{u}(\cdot, t)\|_{L^{2}(\mathbf{R})}^{2 / 3} d t
\end{gathered}
$$


Since, by Hölder's inequality, we have

$$
\int_{0}^{T}\|D \boldsymbol{u}(\cdot, t)\|_{\ell^{2}(\mathbf{R})}^{2 / 3} d t \leq 2^{2 / 3}\left\{(1+T) \int_{0}^{T}(1+t)\|D \boldsymbol{u}(\cdot, t)\|_{\ell^{2}(\mathbf{R})}^{2} d t\right\}^{1 / 3}
$$

we see thad, selting

$$
E(T) \equiv(1+T)\|\boldsymbol{u}(\cdot, T)\|_{L^{2}(\mathbf{R})}^{2}+\int_{0}^{T}(1+l)\|D \boldsymbol{u}(\cdot, l)\|_{L^{2}(\mathbf{R})}^{2} d l
$$

we get, from $(20)$,

$$
E(T) \leq C_{\mathcal{K}}\left\{1+(1+T)^{1 / 3} E(T)^{1 / 3}\right\}
$$

for some constant $C_{\mathcal{K}}$, which depends on $\mathcal{K}=\{m, K, \mu\}$. This immediately gives

$$
E(T) \leq C_{K}(1+T)^{1 / 2}
$$

for some suitable constanil $C_{\mathcal{K}}$ which, agair, deperds on $\mathcal{K}=\{m, K, \mu\}$. Re(alling (21), we them have the following result.

Theorem 1. Let $\boldsymbol{u}(x, t)$ be the solution of (1)-(3) corresponding to an initial profile $\boldsymbol{u}(\cdot, 0)$ in $L^{1}(\mathbf{R}) \cap L^{2}(\mathbf{R})$. Then, there cxists a constant $C_{X}$ (depending on the set of parameters $\mathcal{K}=\{m, K, \mu\}$ given in (2), (13)) such that

$$
\begin{aligned}
& (1+T)\|\boldsymbol{u}(\cdot, T)\|_{h^{2}(\mathbf{R})}^{2}+\int_{0}^{T}(1+t)\|D \boldsymbol{u}(\cdot, t)\|_{h^{2}(\mathbf{R})}^{2} d t \leq C_{\mathcal{K}}(1+T)^{1 / 2} \\
& \text { for exery } T>0 .
\end{aligned}
$$

In particular, this shows (5). As mentioned in the previous section, we can extend the above analysis to the slightly more general equation

$$
\boldsymbol{u}_{t}+(\boldsymbol{u} \varphi(\boldsymbol{u}))_{x}=\left(B(u) \boldsymbol{u}_{x}\right)_{x}
$$

where $B(\boldsymbol{u})$ salisfies (2), (3) and $\varphi$ now depends more generally on the conserved variables $\boldsymbol{u}$ instead of their magnitude $|\boldsymbol{u}|$ only. Observe that, changing the variable $x$ to $\xi=x-\varphi(0) t$ if necessary, we may assume without any loss of 
generality that $\varphi(\mathbf{0})=0$. I'hus, we will make the assumption that, for all $\boldsymbol{u}$ concerned, we have

$$
|\varphi(\boldsymbol{u})| \leq \boldsymbol{\boldsymbol { u }} \mid
$$

for some constant $\Phi>0$. As before, the initial state $\boldsymbol{u}(\cdot, 0)$ is taken in the space $I^{1}(\mathbf{R}) \cap I^{2}(\mathbf{R})$, and we let $K>0$ be large enough so that

$$
\|\boldsymbol{u}(\cdot, 0)\|_{1,2(\mathbf{R})} \leq K
$$

Multiplying the $i^{\text {th }}$ component of cquation (23) by $(1+t) u_{i}(x, t)$ and integrating the result over $\mathbf{R} \times[0, T]$, we then obtain, summing from $i=1$ to $m$,

$$
\begin{aligned}
(1+ & T)\|\boldsymbol{u}(\cdot, T)\|_{L^{2}(\mathbf{R})}^{2}+2 \mu \int_{0}^{T}(1+l)\|D \boldsymbol{u}(\cdot, l)\|_{T^{2}(\mathbf{R})}^{2} d t \\
& \leq\|\boldsymbol{u}(\cdot, 0)\|_{L^{2}(\mathbf{R})}^{2}+\int_{0}^{T}\|\boldsymbol{u}(\cdot, t)\|_{L^{2}(\mathbf{R})}^{2} d t \\
& +2 \int_{0}^{t}(1+l) \int_{-\infty}^{+\infty}\left(\boldsymbol{u}, \boldsymbol{u}_{m}\right) \varphi(\boldsymbol{u}) d x d l
\end{aligned}
$$

where we have used (2). Since, for $\mu, \Phi$ given in (2), (24) above, we have

$$
\begin{gathered}
\int_{-\infty}^{+\infty}\left|\left(\boldsymbol{u}, \boldsymbol{u}_{x}\right)\right||\varphi(\boldsymbol{u})| d x \leq \\
\leq \frac{\Phi^{2}}{2 \mu}\|\boldsymbol{u}(\cdot, t)\|_{L^{4}(\mathbf{R})}^{4}+\frac{\mu}{2}\|D \boldsymbol{u}(\cdot, t)\|_{L^{2}(\mathbf{R})}^{2}
\end{gathered}
$$

we sec that (26) yichds

$$
\begin{aligned}
\left(1+T^{\prime}\right)\|\boldsymbol{u}(\cdot, T)\|_{L^{2}(\mathbf{R})}^{2}+\mu \int_{0}^{T}(1+t)\|D \boldsymbol{u}(\cdot, t)\|_{L^{2}(\mathbf{R})}^{2} d t \\
\quad \leq\|\boldsymbol{u}(\cdot, 0)\|_{L^{2}(\mathbf{R})}^{2}+\int_{0}^{T}\|\boldsymbol{u}(\cdot, l)\|_{L^{2}(\mathbf{R})}^{2} d l \\
\quad+\frac{\Phi^{2}}{\mu} \int_{0}^{T}(1+t)\|\boldsymbol{u}(\cdot, t)\|_{L^{1}(\mathbf{R})}^{1} d t
\end{aligned}
$$

Using the Sobolev inequality

$$
\|\boldsymbol{u}(\cdot, t)\|_{L^{4}(\mathbf{R})}^{2} \leq C_{m}^{i}\|\boldsymbol{u}(\cdot, t)\|_{L^{1}(\mathbf{R})}\|D \boldsymbol{u}(\cdot, t)\|_{L^{2}(\mathbf{R})}
$$


we then obtain, from $(26)^{\prime}$,

$$
\begin{aligned}
(1+ & T)\|\boldsymbol{u}(\cdot, T)\|_{L^{2}(\mathbf{R})}^{2}+\mu \int_{0}^{T}(1+l)\|D \boldsymbol{u}(\cdot, l)\|_{L^{2}(\mathbf{R})}^{2} d t \\
& \leq\|\boldsymbol{u}(\cdot, 0)\|_{L^{2}(\mathbf{R})}^{2}+\int_{0}^{T}\|\boldsymbol{u}(\cdot, t)\|_{T^{2}(\mathbf{R})}^{2} d t \\
& +\frac{\Phi^{2}}{\mu} C_{m}^{2} \int_{0}^{T}(1+t)\|\boldsymbol{u}(\cdot, t)\|_{L^{1}(\mathbf{R})}^{2}\|D \boldsymbol{u}(\cdot, t)\|_{L^{2}(\mathbf{R})}^{2} d t
\end{aligned}
$$

Since (15) remains valid for equation (23), we then oblain, from (13), (25), (28),

$$
\begin{aligned}
(1+ & T)\|\boldsymbol{u}(\cdot, T)\|_{L^{2}(\mathbf{R})}^{2}+\int_{0}^{T}(1+t)\|D \boldsymbol{u}(\cdot, t)\|_{L^{2}(\mathbf{R})}^{2} d t \\
& \leq C_{\mu, K}\left\{1+\int_{0}^{T}\|\boldsymbol{u}(\cdot, t)\|_{L^{2}(\mathbf{R})}^{2} d t\right\}
\end{aligned}
$$

provided we assume that $\|\boldsymbol{u}(\cdot, 0)\|_{L_{J^{\prime}(\mathbf{R})}}$ is small enough to satisfy

$$
\frac{\Phi}{\mu} C_{m}\|\boldsymbol{u}(\cdot, 0)\|_{L^{1}(\mathbf{R})} \leq \frac{1}{2}
$$

where $\mu, \Phi, C_{m}>0$ are the comstants given in (2), (24), (27). Proceeding with (29) as wo did with (20) above, we then get the following result.

Theorem 2. Let $\boldsymbol{u}(x, t)$ be the solution of (23) corresponding to an inilial profile $\boldsymbol{u}(\cdot, 0)$ in the space $L^{1}(\mathbf{R}) \cap L^{2}(\mathbf{R})$. Then, there exists $\delta>0$ (depending on $\mu, \Phi, C_{m}$ given in (2), (24), (27)) such that, whenever $\|\boldsymbol{u}(\cdot, 0)\|_{L^{1}(\mathbf{R})} \leq \delta$, one has

$$
(1+T)\|\boldsymbol{u}(\cdot, T)\|_{L^{2}(\mathbf{R})}^{2}+\int_{0}^{T}(1+l)\|D \boldsymbol{u}(\cdot, l)\|_{L^{2}(\mathbf{R})}^{2} d l \leq C_{K}(1+T)^{1 / 2}
$$

for every $T>0$, where $Q_{K}$ is a postive conslant which depends on the set of parameters $\mathcal{K}=\{m, K, \mu\}$ given in (2), (25).

\section{Multidimensional systems}

In this section, we will extend the analysis above 1.o multidimensional systems of the lorm

$$
\frac{\partial \boldsymbol{u}}{\partial t}+\sum_{k=1}^{N} \frac{\partial}{\partial x_{k}}\left(\boldsymbol{u} \varphi_{k}(|\boldsymbol{u}|)\right)=\sum_{k=1}^{N} \frac{\partial}{\partial x_{k}}\left(B^{[k]}(\boldsymbol{u}) \frac{\partial \boldsymbol{u}}{\partial x_{k}}\right)
$$


where $\varphi_{1}, \ldots, \varphi_{N}$ are given scalar functions, $\boldsymbol{u}(\boldsymbol{x}, t)=\left(u_{1}(\boldsymbol{x}, t), \ldots, u_{m}(\boldsymbol{x}, t)\right)$ is the vector of unknowns, $\boldsymbol{x}=\left(x_{1}, \ldots, x_{N}\right)$, and $B^{[k]}(\boldsymbol{u}), k=1, \ldots, N$, are positive definite matrices of order $m$ satisfying (10), (11) for all $\boldsymbol{u}$ concerned, i.e.,

$$
\left(\boldsymbol{\xi}, B^{[k]}(\boldsymbol{u}) \boldsymbol{\xi}\right) \geq \mu|\boldsymbol{\xi}|^{2} \quad \forall \boldsymbol{\xi} \in \mathbf{R}^{m}, k=1, \ldots, N
$$

and

$$
\left|b_{i, j}^{[k]}(\boldsymbol{u})\right| \leq C\left|u_{i}\right| \quad \forall i, j=1, \ldots, m, i \neq j, k=1, \ldots, N
$$

for some positive constants $\mu, C$, where $b_{i j}^{[k]}(\boldsymbol{u}), i, j=1, \ldots, m$, denote the elements of $B^{[k]}(\boldsymbol{u}), k=1, \ldots, N$. One then obtain

$$
\|\boldsymbol{u}(\cdot, t)\|_{L^{1}\left(\mathbf{R}^{N}\right)} \leq\|\boldsymbol{u}(\cdot, 0)\|_{J^{1}\left(\mathbf{R}^{N}\right)} \quad \forall t>0
$$

which can be shown in the same way as (15) above. In fact. it is straightforward to extend the one-dimensional analysis of the previous section to the N-dimensional equation (31), so that we will only give here a brief sketch of the argument in this case. To get the appropriate decay rates, we multiply the $i^{\text {th }}$ component of $(31)$ by $(1+t)^{N} u_{i}(\boldsymbol{x}, t)$ and integrate the result over $\mathbf{R}^{N} \mathrm{x}[0, T]$ to get, summing from $i=1$ to $m$,

$$
\begin{gathered}
(1+T)^{N}\|\boldsymbol{u}(\cdot, T)\|_{L^{2}\left(\mathbf{R}^{N}\right)}^{2}+2 \mu \int_{0}^{T}(1+t)^{N}\|D \boldsymbol{u}(\cdot, t)\|_{L^{2}\left(\mathbf{R}^{N}\right)}^{2} d t \\
\leq\|\boldsymbol{u}(\cdot, 0)\|_{L^{2}\left(\mathbf{R}^{N}\right)}^{2}+N \int_{0}^{T}(1+t)^{N-1}\|\boldsymbol{u}(\cdot, t)\|_{L^{2}\left(\mathbf{R}^{N}\right)}^{2} d t
\end{gathered}
$$

where

$$
\|D \boldsymbol{u}(\cdot, t)\|_{L^{2}\left(\mathbf{R}^{N}\right)}^{2}=\sum_{i=1}^{m} \sum_{j=1}^{N}\left\|\frac{\partial u_{i}}{\partial x_{j}}(\cdot, t)\right\|_{L^{2}\left(\mathbf{R}^{N}\right)}^{2}
$$

Using (34) and the Sobolcy incquality

$$
\|\boldsymbol{u}(\cdot, t)\|_{L^{2}\left(\mathbf{R}^{N}\right)} \leq C_{m, N}\|\boldsymbol{u}(\cdot, t)\|_{L^{1}\left(\mathbf{R}^{N}\right)}^{\frac{2}{N+2}}\|D \boldsymbol{u}(\cdot, t)\|_{L^{2}\left(\mathbf{R}^{N}\right)}^{\frac{N}{N+2}}
$$

we get.

$$
\|\boldsymbol{u}(\cdot, l)\|_{L^{2}\left(\mathbf{R}^{N}\right)} \leq C_{m, N, K}\|D \boldsymbol{u}(\cdot, l)\|_{L^{2}\left(\mathbf{R}^{N}\right)}^{\frac{N}{N+2}}
$$


where $C_{m, N, K}$ denotes a constant which depends on $m, N$ and $K>0$ such that

$$
\|\boldsymbol{u}(\cdot, 0)\|_{L^{1}\left(\mathbf{R}^{N}\right)} \leq K \quad: \quad\|\boldsymbol{u}(\cdot, 0)\|_{L^{2}\left(\mathbf{R}^{N}\right)} \leq K
$$

Hence, (35) gives

$$
\begin{aligned}
& (1+T)^{N}\|\boldsymbol{u}(\cdot, T)\|_{L^{2}\left(\mathbf{R}^{N}\right)}^{2}+2 \mu \int_{0}^{T}(1+l)^{N}\|D \boldsymbol{u}(\cdot, l)\|_{L^{2}\left(\mathbf{R}^{N}\right)}^{2} d l \\
& \quad \leq\|\boldsymbol{u}(\cdot, 0)\|_{L^{2}\left(\mathbf{R}^{N}\right)}^{2}+C_{m, N, K} \int_{0}^{T}(1+l)^{N-1}\|D \boldsymbol{u}(\cdot, l)\|_{L^{2}\left(\mathbf{R}^{N}\right)}^{\frac{2 N}{N+2}} d l
\end{aligned}
$$

where $C_{m, N, K}$ denotes a constant which depends on $m, N, K$. Since

$$
\begin{aligned}
& \int_{0}^{T}(1+t)^{N-1}\|D \boldsymbol{u}(\cdot, t)\|_{L^{2}\left(\mathbf{R}^{N}\right)}^{\frac{2 N}{N+2}} d t \leq \\
& \quad \leq\left(\frac{2}{N}\right)^{\frac{2}{N+2}}\left\{(1+T) \int_{0}^{T}(1+l)^{N}\|D \boldsymbol{u}(\cdot, l)\|_{L^{2}\left(\mathbf{R}^{N}\right)}^{2} d t\right\}^{\frac{N}{N+2}}
\end{aligned}
$$

we then get, from (38),

$$
\begin{aligned}
& (1+T)^{N}\|\boldsymbol{u}(\cdot, T)\|_{\ell^{2}\left(\mathbf{R}^{N}\right)}^{2}+\int_{0}^{T}(1+t)^{N}\|D \boldsymbol{u}(\cdot, t)\|_{L^{2}\left(\mathbf{R}^{N}\right)}^{2} d t \\
& \leq G_{K}\left\{1+(1+T)^{\frac{N}{N+2}}\left(\int_{0}^{T}(1+t)^{N}\|D \boldsymbol{u}(\cdot, t)\|_{L^{2}\left(\mathbf{R}^{N}\right)}^{2} d t\right)^{\frac{N}{N+2}}\right\}
\end{aligned}
$$

where $C_{\mathcal{K}}$ is some positive constant which depends on the set of parameters $\mathcal{K}=\{m, n, K, \mu\}$. Proceeding as in (20)-(22), we then immediately obtain the following resul1.

Theorem 3. Let $\boldsymbol{u}(\boldsymbol{x}, 1)$ be the solution of equation (31)-(33) corresponding to an initial state $\boldsymbol{u}(\cdot, 0)$ in the space $L^{1}\left(\mathbf{R}^{N}\right) \cap L^{2}\left(\mathbf{R}^{N}\right)$. Then, there cxists a positive constant $C_{\mathcal{K}}$ (depending on $\mathcal{K}=\{m, N, K, \mu\}$ given in (32), (37)) such that

$$
(1+T)^{N}\|\boldsymbol{u}(\cdot, T)\|_{L^{2}(\mathbf{R})}^{2}+\int_{0}^{T}(1+t)^{N}\|D \boldsymbol{u}(\cdot, t)\|_{L^{2}(\mathbf{R})}^{2} d t \leq C_{K}(1+T)^{N / 2}
$$

for enery $T>0$. 


\section{References}

[1] Brio, ..; Hunter; J. K., Rotalionally invarianl hyperbolic waves, Comm. Purc Appl. Ma1h. 13, (1990), 1037-1053.

[2] Brio, M.; Hunter, J. K., Nonlinear transverse waves in an isotropic medium, Third Intermational Conference on IIyperbolic Problems, Uppsala, 1990, $131-140$.

[3] Cherr, I. T., Mulliple-mode diffusion waves for viscous non-slriclly hyperbolic conservalion laws, Comm. Math. Phys. 138, (1991). 51-61.

[4] Chern, I. L.; Liu, T. P., Convergence to Diffusion Waves of Solutions for Viscous Conservation Laws, Comm. Math. Phys. 110, (1987), 503-517 \& $120,(1989), 525-527$.

[5] Freistühler, H., On compact linear degeneracy. IMA preprint series \# 551, August 1989.

[6] Freistühler, H., Rotational degeneracy of hyperbolic systems of conservation laws, Arch. Rat. Mech. Anal. 113, (1990), 39-64.

[7] Isaacson, E., Global solution of a Riemann Problem for a non-strictly hyperbolic system of conservation laws arising in enhanced oil recovery. Rockefeller University preprints, 1982.

[8] Kawashima, S., Large-time behaviors of solutions to hyperbolic-parabolic systems of conservation laws and applications, Proc. Roy. Soc. Edinburgh $106 \mathrm{~A},(1987), 169-194$.

[9] Keyfitz, B. L.; Kranzer, H. C., A system of non-strictly hyperbolic conservation laws arising in elasticity theory, Arch. Rat. Mech. Anal. 72, (1980), 219-241.

[10] Kreiss, H. O.; Lorenz, J., Initial-Boundary Value Problems and the NavierStokes Fqualions, Academic Press, New York, 1989. 
[11] Ladyzhenskaya, O. A.; Solonnikov, V. A.; Ural'ceva, N. N., Linear and Quasi-linear Equations of Parabolic 'lype, American Mathematical Society, Providence, 1968.

[12] Temple, B., Global solution of the Cauchy problem for a class of $2 \times 2$ nonstrictly hyperbolic conservation laws, Adv. Appl. Math. 3, (1982), $335-375$.

[13] Zingano, P. R., Diffusion waves of a rotationally invariant parabolic system. Bol. Soc. Bras. Mat. Aplic. Comput. 5. (1994), 51-б̌6.

[14] Zingano, P. R., Nonlinear slability of rarefaction waves for a scalar conservation law with viscosity. (submitted)

[15] 7ingano, P. R., Mullidimensional diffusion waves. (submitied)

Departamento de Matemática Pura e Aplicada

Instituto de Matemática

Universidade Federal do Rio Grande do Sul

Porto Alcgre, RS 91509-900

Brazil

Fax: 55-51-3191512

E-mail: zingano(i)mat.ufrgs.br 